\title{
Targeting energy expenditure via fuel switching and beyond
}

\author{
J. G. Geisler
}

Received: 28 May 2010 /Accepted: 19 August 2010/Published online: 17 October 2010

(C) Springer-Verlag 2010

\begin{abstract}
Since over-nutrition accelerates the development of obesity, progression to type 2 diabetes, and the associated co-morbidity and mortality, there has been a keen interest in therapeutic interventions targeting mechanisms that may curb appetite, increase energy expenditure or at least attenuate insulin resistance. Over the past decade, numerous peri-mitochondrial targets in the de novo lipid synthesis pathway have been linked to an increase in energy expenditure and the drug development industry has pursued the gene products involved as candidates to develop drugs against. The basis of this link, and specifically the premise that lowering tissue and cellular malonyl-CoA can increase energy expenditure, is scrutinised here. The argument presented is that fuel switching as effected by changes in cellular malonyl-CoA concentrations will not trigger the mitochondria to increase energy expenditure because: (1) an increase in beta-oxidation by lowering respiratory exchange ratio (indicative of the metabolic fuel consumed) does not equal an increase in energy expenditure (how rapidly fuel is consumed); (2) the ATP:oxygen ratios (i.e. ATP energy made:oxygen required for the reaction) are similar when metabolising lipids (2.8) vs glucose (3.0); (3) substrate availability (NEFA) does not drive energy expenditure in vivo; and (4) the availability of ADP in the mitochondrial matrix determines the rate of energy expenditure, not the availability of fuel to enter the mitochondrial matrix. To increase mitochondrial energy expenditure, work must be done (exercise) and/or the mitochondrial proton leak must be enhanced, both of which
\end{abstract}

J. G. Geisler $(\bowtie)$

Johnson \& Johnson Pharmaceutical Research and Development,

L.L.C., Metabolic Disease, Drug Discovery,

Welsh \& McKean Roads,

Spring House, PA 19477, USA

e-mail: Jgeisle1@its.jnj.com increase availability of ADP. In fact, despite the historic taboo of chemical uncoupling, this mechanism validated in humans is closest on task to increasing whole-body energy expenditure. Chemical uncoupling mimics the naturally occurring phenomenon of proton leak, accelerating the metabolism of glucose and lipids. However, it is completely non-genomic (i.e. the target is a location, not a gene product) and is not associated with addiction or mood alterations common to satiety agents. A significant hurdle for drug development is to discover a safe mitochondrial uncoupler and to formulate it potentially as a pro-drug and/or oral pump, to avoid the issue of overdosing experienced in the 1930s. The potential therapeutic impact of such a compound for an overnutritioned patient population could be profound. If effective, the mitochondrial uncoupler mechanism could resolve many of the associated diseases such as type 2 diabetes, hypertension, obesity, depression, sleep apnoea, non-alcoholic steatohepatitis, insulin resistance and hyperlipidaemia, therefore becoming a 'disease-modifying therapy'.

Keywords $\beta$-Oxidation - Brown adipose tissue $\cdot$ Chemical uncoupling $\cdot$ Disease-modifying therapy $\cdot$ Energy expenditure $\cdot$ Insulin resistance $\cdot$ Malonyl-CoA .

Mitochondria - Obesity Type 2 diabetes

$\begin{array}{ll}\text { Abbreviations } \\ \text { ACC } & \text { Acetyl-coenzyme A carboxylase } \\ \text { BAT } & \text { Brown adipose tissue } \\ \text { BMR } & \text { Basal metabolic rate } \\ \text { CPT1A } & \text { Carnitine palmitoyltransferase-1A } \\ \mathrm{KO} & \text { Knockout } \\ \mathrm{P}: \mathrm{O} & \text { ATP:oxygen } \\ \mathrm{RER} & \text { Respiratory exchange ratio } \\ \mathrm{UCP1} & \text { Uncoupling protein 1 } \\ \dot{V} \mathrm{O}_{2} & \text { Volume of oxygen consumed }\end{array}$




\section{The premise}

In the last decade, seminal papers have been published in which gene deletion of targets peripheral to the mitochondria resulted in increased beta-oxidation and weight loss. For example, stearoyl-coenzyme A desaturase 1 null mutation was reported to protect against adiposity and increased fatty acid oxidation [1]. Subsequently, it was understood that this gene also had an essential role within the dermis and the animals were hypothermic at room temperature [2]. Similarly, diacylglycerol $O$-acyltransferse2 has a role in providing a skin barrier [3]. These enzymes, as well as acetyl-coenzyme A carboxylase (ACC) 1 and 2 [4], fatty acid synthetase [5] and mitochondrial glycerol-3phosphate acyltransferase-1 [6], are key enzymes in the de novo synthesis of triacylglycerol. Of central interest are ACC1 and 2, since they directly generate malonyl-CoA, which plays a dual role in the liver [4]. Malonyl-CoA donates the first two carbons for de novo synthesis of palmitate and binds to carnitine palmitoyltransferase-1a (CPT1A) as an allosteric inhibitor of fatty acid transport into the mitochondrial matrix. This second role interested pharmacologists because it was believed that lowering cellular malonyl-CoA would result in increased betaoxidation of lipids and increased energy expenditure in the mitochondria. Why did we believe this hypothesis? First, malonyl-CoA allosterically inhibits CPT1A. Second, malonyl-CoA levels drop in the fasted state and subsequently beta-oxidation of fatty acids begins. Third, the knockout (KO) of $A c c 2$ (also known as $A c a c b$ ) resulted in increased energy expenditure as measured by the volume of oxygen consumed $\dot{V} \mathrm{O}_{2}[7,8]$. It has also been shown that inhibition of the downstream genes $S c d 1$ and Dgat2 also lowers $A c c 1$ (also known as Acaca) and Acc2 expression by a feedback mechanism [9-11]. These results supported drug research targeted approaches to lower malonyl-CoA. However, there are several problems with this premise, including the unusual phenotype of $A c c 2 \mathrm{KO}$ mice. First, it is unlikely that lowering malonyl-CoA's allosteric inhibition of CPT1A in the fed state will result in enhanced oxidation of lipids, with concomitant weight reductions and improved insulin sensitivity. Second, the first Acc2 KO animals did not show a switching of fuels (i.e. change in respiratory exchange ratio [RER]), but rather an increase in $\dot{V} \mathrm{O}_{2}$, which was not predicted by bioenergetics or evident with a dual Acc1 and Acc2 inhibitor in rats [12]. Recently, the Acc2 KO model was independently generated and resulted in an opposite phenotype [13]. The regenerated Acc2 KO animals had lower malonyl-CoA levels, lower RER and increased fatty acid oxidation, but there was no change in $\dot{V} \mathrm{O}_{2}$, adiposity and body weight, or more importantly no improvements in glycaemic control during a glucose tolerance test on a low- or high-fat diet [13]. The premise that purely switching metabolism from glucose to lipid oxidation by lowering malonyl-CoA will result in increased energy expenditure and be useful in treatment of metabolic disease is clearly unsupported. In fact, it may exacerbate the phenotype.

Further down the de novo lipid synthesis pathway, interesting observations have been made in the phenotype of mitochondrial Gpat1 (also known as Gpam) KO mice on a high-fat diet [14]. Glycerol-3-phosphate acyltransferase-1 catalyses the initial and rate-limiting step for addition of the first long-chain acyl-CoA on to the glycerol-3-phosphate backbone to partition lipids towards synthesis of triacylglycerol and away from degradative pathways. The longterm consequence of placing the KO phenotype on a highfat diet was increased insulin resistance. Gpat1 KO mice had increased hepatic acyl-carnitines, a measurement of the abundance of partially processed fatty acids leaving the mitochondria and diffusing into the plasma compartment. The conclusion drawn from the Gpat1 KO studies was that 'the amount of acyl-CoA exceeded the capacity of the mitochondrial oxidation pathway' [14]. The effect may be real, but the conclusion is inaccurate. Instead, it is possible that lowering malonyl-CoA without an increase in energy expenditure was the cause of increased acyl-CoA levels. It is unlikely that the mitochondrial machinery was overwhelmed by an excess of lipids to metabolise. It is, rather, more likely that the mitochondria could not metabolise more lipids because ATP levels were sufficient and ADP levels insufficient. Indeed, if these mice had been exercising or administered a mitochondrial uncoupler such as 2,4dinitrophenol, it is possible that the mitochondria would have metabolised the back-log of acyl-CoAs, thus restoring sufficient levels of ADP.

\section{Energy expenditure is dependent on ADP availability and not substrate availability}

A widely held but false presumption that seems to act as a dividing line between the mitochondrial bioenergetic and the diabetes/obesity communities is that increasing substrate into the mitochondria will increase energy expenditure. In contrast to experiments on isolated mitochondria or in vitro studies [15], the infusion of lipids in humans did not change energy expenditure [16], but did increase insulin resistance [17]. If the allosteric block (malonyl-CoA) is removed from CPT1A, fatty acids will enter the mitochondria, but do not become oxidised until energy is needed. The ability to get into the mitochondria is a necessary condition, but not in itself sufficient for the oxidation of lipids. The metabolism of lipids is dependent on the availability of ADP [18], not on whether fatty acids can get into the mitochondria. If most of the ADP is locked up 
as ATP, the condition typically found in the fed state, then intra-mitochondrial lipids will not be used. Mitochondrial toxicity or insulin resistance may arise if all normal functions (e.g. oxidative phosphorylation, electron transport, citric acid cycle) are to continue against a background of lipid overabundance [14]. Although oxidation of NEFA can be induced reproducibly in vitro [15], there is no evidence that increasing substrate in vivo will increase energy expenditure. Increasing substrate in humans has been shown to increase insulin resistance and fat storage $[17,19]$. The function of CPT1A is not to block or modulate the rate of fatty acid oxidation, but rather to regulate lipid substrate availability into the inner mitochondrial matrix, thus governing against an overabundance of intra-mitochondrial fatty acids. The use of an in vitro beta-oxidation assay to select targets and compounds in order to suggest that the same effect occurs in vivo can potentially lead to incorrect conclusions. Evidence of increased energy expenditure must be demonstrated experimentally (e.g. $\dot{V} \mathrm{O}_{2}$, tracers) in an intact animal.

The phenomenon of adaptive increases in energy expenditure in response to energy intake and weight gain is evident in rodents [20], but perhaps to a considerable less degree in humans. It has been clearly demonstrated in a head-to-head comparison of the Ucpl KO mouse with a wild-type mouse during which both were fed a high-fat diet and housed at thermoneutrality, i.e. $\sim 30^{\circ} \mathrm{C}\left(82^{\circ} \mathrm{F}\right)$, when non-shivering thermogenesis is turned off. Both animals gained considerable weight relative to when housed at room temperature $\left(25^{\circ} \mathrm{C}\right)[21]$, but at $\sim 30^{\circ} \mathrm{C}$ the wild-type mouse gained far less weight than its Ucp1 KO counterpart [22]. At this temperature, the sympathetic nervous system should not stimulate proton leak via uncoupling protein 1 (UCP1) in a fully 'clothed mouse' [23] and yet a compensatory effect of resistance to weight gain due to the high energy diet was still observed [24]. It is unlikely that such a mechanism will provide a meaningful impact in humans consuming high-fat diets (i.e. a diet-induced increase in activity and/or thermogenesis), since (1) the mechanism appears to be mediated by UCP1, (2) brown adipose tissue (BAT) mass is considerably lower and (3), more importantly, we have a pandemic of obesity.

\section{Fuel switching does not increase energy expenditure}

A second possible misconception is that switching fuels will increase oxidation and this in turn will increase energy expenditure. When malonyl-CoA is reduced, the NADH/ $\mathrm{FADH}_{2}$ needed to drive the electron transport chain and subsequently oxidative phosphorylation is forced to be derived from NEFA by beta-oxidation and from the remaining acetyl-CoAs by the citric acid cycle vs metabolism of glucose (Fig. 1). However, regardless of whether the source of

Glucose

Lipids

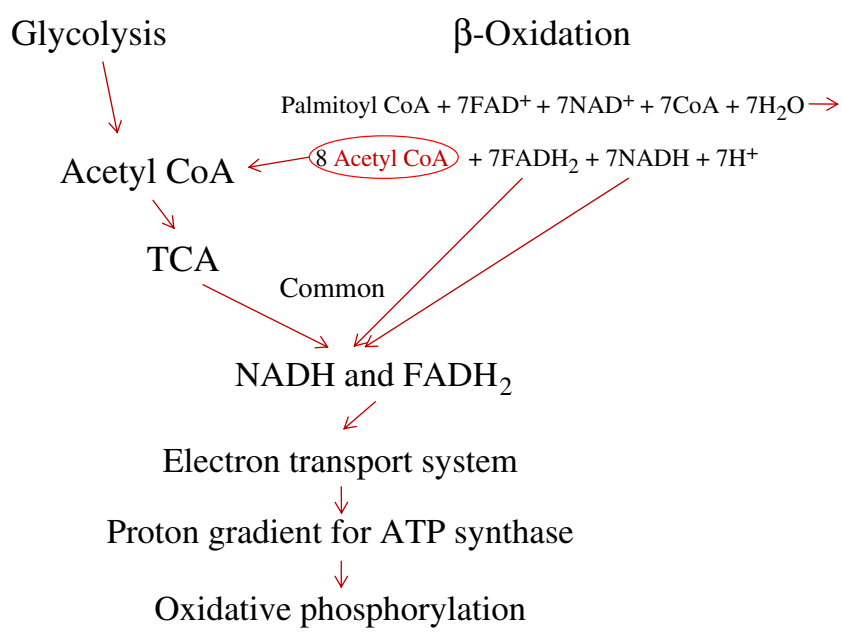

Fig. 1 Metabolism of glucose and lipids is the source of the proton gradient $\left(\Delta \widetilde{u}_{\mathrm{H}^{+}}\right)$. NADH and $\mathrm{FADH} \mathrm{H}_{2}$ derived from the metabolism of glucose and lipids provide the protons used to maintain the proton gradient across the mitochondrial matrix

$\mathrm{NADH} / \mathrm{FADH}_{2}$ is from glucose or lipid, the ATP:oxygen (P:O) ratio (ratio of ATP produced to the amount of oxygen required and consumed for the reaction) remains roughly equivalent (Fig. 2). The metabolism of 1 mole of glucose consumes 12 moles of oxygen and yields 36 moles of ATP. Therefore, the $\mathrm{P}: \mathrm{O}$ ratio for glucose is $36 / 12=3$. Metabolism of 1 mole of palmitate requires 45.7 moles of $\mathrm{O}_{2}(16 / 0.7 \times 2)$ and yields 129 moles of ATP. Therefore, the P:O ratio is 129/ $45.7=2.8$ [25]. This implies that no more energy is gained or lost simply by switching fuel, and therefore the mitochondria's energy balance remains equal until the rate of oxygen consumption changes (energy expenditure). This was clearly demonstrated in rats administered a dual $\mathrm{ACC} 1$ and $\mathrm{ACC} 2$ inhibitor or in the recent $A c c 2 \mathrm{KO}$ model, where RER was constitutively lowered (as expected), but $\dot{V} \mathrm{O}_{2}$ consumed remained unchanged $[12,13]$. The reason why $\dot{V} \mathrm{O}_{2}$ was unaltered is simply that switching fuels does not motivate the mitochondria to increase energy expenditure. The P:O ratios are equivalent, and therefore ATP levels or mitochondrial

Glucose

$$
\begin{array}{cc}
\mathrm{C}_{6} \mathrm{H}_{12} \mathrm{O}_{6}+6 \mathrm{O}_{2} \rightarrow 6 \mathrm{CO}_{2}+6 \mathrm{H}_{2} \mathrm{O} & \mathrm{C}_{16} \mathrm{H}_{32} \mathrm{O}_{2}+23 \mathrm{O}_{2} \rightarrow 16 \mathrm{CO}_{2}+16 \mathrm{H}_{2} \mathrm{O} \\
\text { Yield: } 36 \mathrm{ATP} & \text { Yield: } 129 \mathrm{ATP}
\end{array}
$$

Fig. 2 ATP:oxygen ratios for different fuels. The ratio of energy yield (ATP) to oxygen required for glucose vs lipid metabolism are roughly equal. Palmitate generates more ATP per molecule, but requires more oxygen to metabolise 
membrane potential remain in balance. It should be noted that although there is a $6.67 \%$ difference between glucose and palmitate $\mathrm{P}: \mathrm{O}$ ratio, it appeared insignificant with regard to providing an advantage in the constitutive fatty acid oxidation state for body weight, glycaemic control or adiposity in $A c c 2 \mathrm{KO}$ animals under high-fat diet conditions.

\section{Animal models with dermis issues}

The heat generated from brown fat (via UCP1) in rodents may also be linked to the possible misinterpretation of the metabolic phenotypes of mouse mutations and compounds that have been reported to reduce body weight in rodents. In rodents, BAT has the capacity to increase energy expenditure $60 \%$ over basal metabolic rate (BMR) through non-shivering thermogenesis [26]. If the function or heat retention capacity of mouse skin is compromised by changing the lipid content, non-shivering thermogenesis is engaged to maintain core body temperature via the sympathetic nervous system [2]. This creates the illusion, on translation to humans when characterising a new target, that the KO phenotype resulted in increased whole body energy expenditure and that the protein may be a suitable target for the development of drugs to treat metabolic disease. Before claiming increased energy expenditure, it should be tested whether the $\mathrm{KO}$ animals or drug candidates can reduce body weight at $30^{\circ} \mathrm{C}$ (thermoneutrality) or when animal models are crossed on to a Ucp1 KO background to eliminate weight reductions due solely to non-shivering thermogenesis [22]. Ideally, compounds should be tested in Ucp 1 $\mathrm{KO}$ animals at $30^{\circ} \mathrm{C}$ to simultaneously eliminate shivering, non-shivering and diet-induced thermogenesis [27]. If greater energy expenditure and weight reductions are still present in the absence of UCP1, then the target or compound tested may well translate into weight loss in humans.

\section{Breaking the taboo of chemical uncoupling}

A significant research effort was undertaken in the 1990s after publication of a seminal paper connecting the phenomenon of non-shivering thermogenesis in BAT to UCP1 [28]. There was a keen interest in developing UCP1 drugs as agonists capable of harnessing the potential of UCP1 to achieve weight loss via energy expenditure mechanisms. However, it was quickly realised that UCP1 is restricted to BAT and adult humans appeared to no longer have this tissue. Only UCP1 has uncoupling properties [29]. These findings led to a steep decline in research on drugs targeting energy expenditure mechanisms and efforts were channelled towards anorectic mechanisms. Recently, BAT was found in humans along the vertebra and shown to be induced upon exposure to cold, presumably also by the sympathetic nervous system $[30,31]$. Expectations of significant weight loss mediated by human BAT should be treated with some caution if the intention is to use the existing tissue or to expand upon the mass of this tissue. One reason for such caution is that humans have proportionally less BAT per body weight than rodents. Current positron emission tomography-computed tomography (PET-CT) scans estimate BAT mass in humans to total $\sim 13 \mathrm{~g}(0.02 \%$ of body weight for a person weighing $70 \mathrm{~kg})$ [32], whereas mouse BAT mass is $\sim 400 \mathrm{mg}$ for $40 \mathrm{~g}$ body weight (1\%) (J.G. Geisler, unpublished results). Therefore, humans have approximately 50 -fold less BAT than mice. More importantly, since humans are thermoneutral [22], it is unlikely that UCP1 could be triggered to uncouple and provide meaningful weight loss at room temperature.

Although there are some 30,000 genes in the genome [33], the most effective means of increasing energy expenditure in humans may be non-genomic (i.e. independent of a gene or protein target), namely chemical uncoupling (i.e. location of the mitochondrial matrix would be the target). There has been renewed interest in chemical uncoupling due to a better understanding of mitochondrial function $[24,34]$ and the challenges of anorectic agents that target reward pathways [35, 36]. Chemical uncouplers mimic the function of UCP1 and the naturally occurring proton leak, which accounts for 20 to $25 \%$ of the energy lost in liver and $\sim 50 \%$ of that lost in muscle, i.e. roughly $25 \%$ of total BMR [37, 38]. Instead of a protein channel for the proton to enter through (e.g. UCP1), the molecule acts as its own proton transporter into the matrix from the cytosol. As a mechanism for effecting weight loss, chemical uncouplers are precisely on task for causing the mitochondria to increase energy expenditure and achieve this by metabolising glucose and NEFA in the process (Fig. 1). The mechanism works as follows. A weakly acidic uncoupler holds a proton that dissociates upon entering the basic environment within the mitochondrial matrix. As a proton has been lost, the molecule leaves the matrix as an anion and, upon returning to the acidic cytosolic environment, becomes reprotonated to a cation, upon which it again returns to the matrix to drop off another proton (Fig. 3). The cumulative effect of such a cycle is a reduction of the mitochondrial membrane potential and a lowering of the proton-motive-force $(\Delta p)$ that would be used to allow a proton to travel through ATP synthase, causing a rotation and subsequent phosphorylation of ADP [39]. Because of this, ATP levels drop and the mitochondria instantly responds to re-establish the gradient by accelerating utilisation of NADH and $\mathrm{FADH}_{2}$. Oxidation is sustained in the presence of an uncoupler because the mitochondrial membrane potential cannot be restored until the drug is cleared/metabolised. These electron transport system sub- 
a
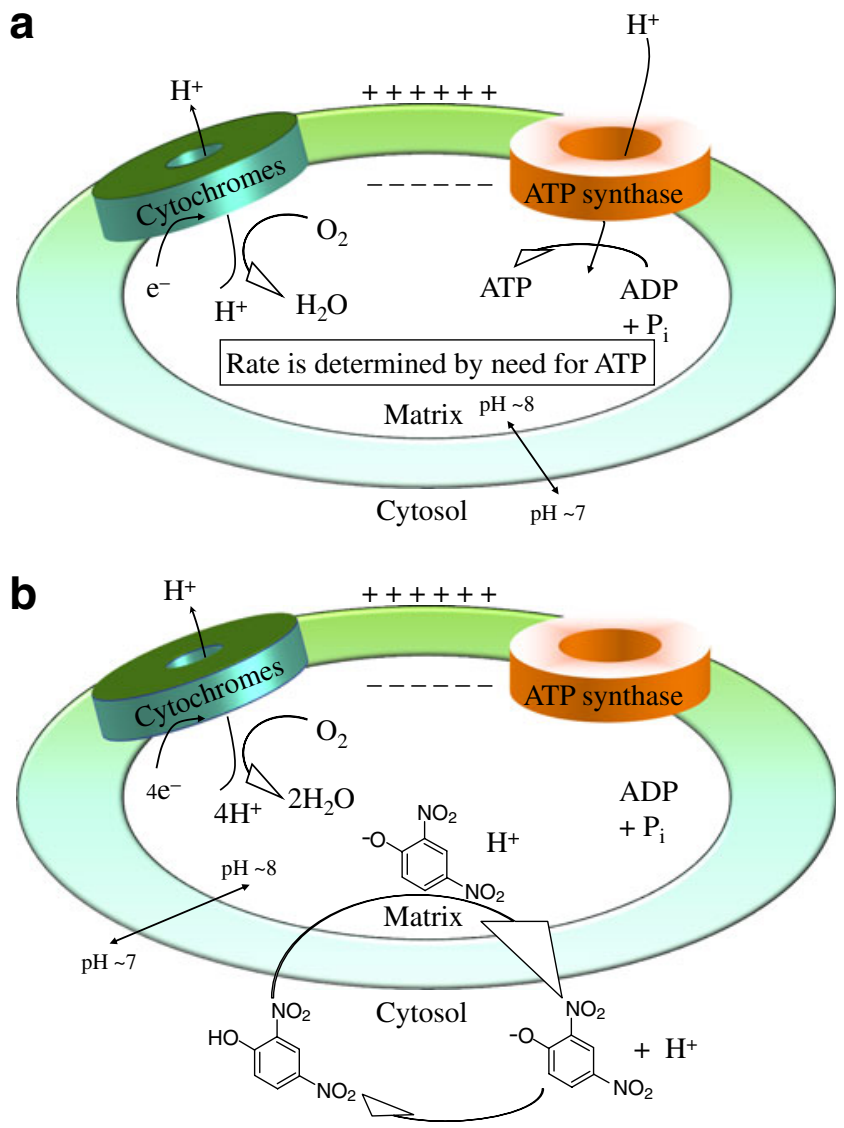

Fig. 3 Coupling vs uncoupling. Maintaining the proton gradient across the mitochondrial matrix and cytosol involves the pumping of protons out of the matrix via cytochromes I, III and IV. a The coupling of a proton transfer to the synthesis of ATP is a result of a proton returning through ATP synthase, causing a rotation and subsequent phosphorylation of ADP, thereby yielding an ATP molecule. b This mechanism is circumvented in the case of chemical uncoupling (i.e. entry of protons without phosphorylation to produce ATP). The proton transfer into the matrix is on a carrier, a weak acid molecule (e.g. 2,4dinitrophenol or carbonyl cyanide $p$-trifluoromethoxyphenylhydrazone). When the weak acid (cation) enters the basic environment of the matrix, a proton is dissociated and the molecule, now an anion, returns to the cytosol to become reprotonated into a cation and start the cycle over again. All mitochondrial systems remain functional, but are accelerated

strates come from two sources, the metabolism of glucose and that of lipids. Energy expenditure is increased as monitored by an increase in oxygen consumed; however, the RER is roughly unchanged and therefore a mixed fuel is consumed. This mechanism motivates the mitochondria towards anti-obesity and anti-diabetes because the proton electrochemical gradient $\left(\Delta \widetilde{u}_{\mathrm{H}^{+}}\right)$is out of balance. This is fundamentally different from malonyl-CoA-lowering mechanisms (Fig. 4). It is important to note that for this mechanism to be efficient, it is critical that all components of the electron transport system, including ATP synthase, remain completely functional. A pure chemical uncoupler is not an inhibitor, but an enhancer of respiration.

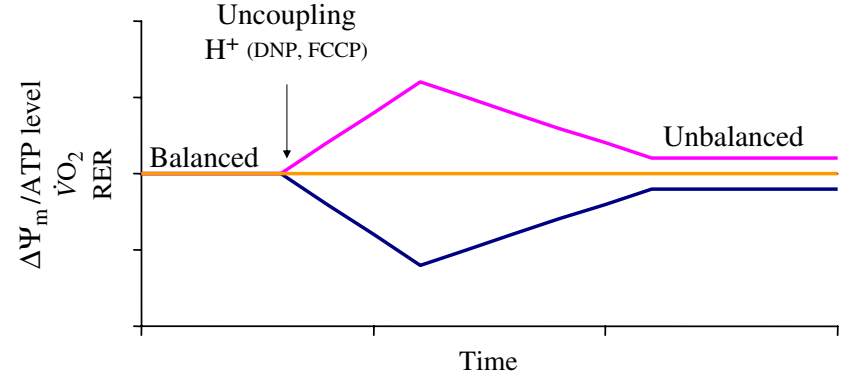

Fig. 4 Dynamics of energy compensation with chemical uncoupling in the fed state. Administration of a chemical uncoupler causes a drop in the mitochondrial membrane potential $\left(\Delta \Psi_{\mathrm{m}}\right)$ and ATP levels (blue line). The cell responds by increasing the rate of oxygen consumption $\left(\dot{V} \mathrm{O}_{2}\right.$ [pink line] or energy expenditure) to generate more NADH and $\mathrm{FADH}_{2}$ as a source of protons (when oxidised back to NAD ${ }^{+}$and FAD) as a mechanism to re-establish the electrochemical gradient $\left(\Delta \widetilde{u}_{\mathrm{H}^{+}}\right)$. Since NADH and $\mathrm{FADH}_{2}$ come from the metabolism of glucose and lipids, the RER (orange line) is primarily unchanged (high doses shift RER towards lipids). In the presence of a chemical uncoupler, energy expenditure is constitutively enhanced since the $\Delta \Psi_{\mathrm{m}}$ is out of balance

Drug safety is a primary concern for the development of chemical uncoupling as a therapeutic approach for metabolic disease. Historical data from the 1930s demonstrate the abuse potential for uncoupler-based weight-loss agents, although 2,4-dinitrophenol was not addictive [40]. Beyond the benefits of weight loss 'predominantly from the hips and abdomen' [40], uncouplers reduce reactive oxygen species [41], a phenomenon that has been associated with improved infarct volume during ischaemia [42], longevity and ageing [43]. Although chemical uncoupling was proven to achieve weight loss in humans during the 1930 s, the science of pharmacology and the challenges of drug development have changed dramatically over the past 80 years. It would need to be demonstrated that all vital functions are uncompromised with chemical uncoupling, in particular the heart in a setting of increased BMR. Another potential risk of chemical uncoupling is increases in core body temperature at high doses, although at lower doses the heat is usually dissipated with no change in temperature [44, 45]. Cataracts have been reported in some patients treated with 2,4-dinitrophenol, but this was not replicated in more recent animal models [46] and may be a property of 2,4-dinitrophenol vs general uncouplers. A key question is to what degree does energy expenditure have to be increased to achieve meaningful weight loss? According to calculations from a study conducted in 1935, a $10 \%$ increase in BMR resulted in weight loss of $\sim 0.3$ to $0.45 \mathrm{~kg} /$ week [44]. If the calculations are accurate and a less aggressive approach was taken today, then a $5 \%$ increase in BMR should result in half that weight loss, but still provide annual weight reductions of $10 \%$ or $\sim 12 \mathrm{~kg}(26 \mathrm{lb})$ for patients with BMI over $30 \mathrm{~kg} / \mathrm{m}^{2}$. Interestingly, there were, 
to the author's knowledge, no reports of a compensatory increase in food consumption due to the increase in energy expenditure in patients treated with 2,4-dinitrophenol in the 1930s [40, 44, 45]. In a recent study, 2,4-dinitrophenol was provided to mice for their entire adult lifespan and daily food consumption remained unchanged. However, a significant reduction in body weight was observed, as well as reduced serum glucose, insulin and triacylglycerol, relative to the untreated group [43]. Over the past 10 years, numerous patents have been published by Novo Nordisk indicating that the company has investigated chemical uncoupling $[47,48]$. To make such an approach pharmaceutically possible, the therapeutic index has to be considerably improved over 2,4-dinitrophenol. Drugs that have the potential to make patients look or feel good are susceptible to abuse. 2,4-Dinitrophenol lacked weight lossdiminishing effects at higher doses, and patients tended to overdose. A modern chemical uncoupler may therefore need to be formulated with a delivery method (e.g. oral pump, pro-drug) that curtails overdosing. The positive and negative long-term consequences of increasing energy expenditure are unknown, but this could be addressed as a component of the drug approval process.

\section{Wasting energy: a unifying theory to tackle over-nutritional phenotypes}

The term 'over-nutritional phenotype' refers to the expressed or displayed effect when energy intake exceeds the tolerated threshold of an individual's combined genetic composition and level of physical activity, shown as an increased incidence of insulin resistance, obesity, type 2 diabetes, cancer, sleep apnoea, depression, inflammation, cardiovascular disease, hypertension, non-alcoholic fatty liver disease, etc. [49-60]. It is critical to understand that even with the most unfortunate genetic composition that predisposes an individual to metabolic disease [61], such as the well documented US Pima Indians with the world's highest prevalence of type 2 diabetes (38\%), that physical activity (energy expenditure) and diet can have a profound effect on upon prevention. As an example, the lesser known Pima Indian tribe in Mexico has a 7\% prevalence of type 2 diabetes, fivefold lower than the US tribe. However, the Mexican Pima Indians are much leaner ( $30 \mathrm{~kg}$ lower body weight), chiefly due to greater physical activity (even in leisure time) and a low-fat diet including complex carbohydrates [62]. Comparative polymorphic analysis suggests that although the US and Mexican tribes are no longer identical, they share a closely held gene pool and that the difference in their phenotype is unlikely to be accounted for by a substantial genetic drift. For the remaining population that find it difficult or impossible to modify diet and increase physical activity, wasting energy (the loss of potential substrates for 'useful work') may be a fundamental mechanism to target over-nutritioned phenotypes (Fig. 5) and should be fully explored. Consider, for example, the energy that is lost by heat via chemical uncoupling vs making ATP or the loss of potential energy through urinary glucose excretion effected by sodium glucose transporter 2 inhibition [63]. These mechanisms create an unbalanced equation in favour of weight loss, without directly targeting pathways controlling mood and satiety. What other 'futile' cycles can be used to change the energy balance? One possibility, perhaps, is the reduction of fatty acid absorption in the intestine or the increase of fatty acid excretion in the urine, making fatty acids thus unavailable as an energy source or as signalling molecules. These ideas, centred on the notion of 'wasting energy', as well as a paradigm shift from the historic focus on searching for risk alleles towards a considerably increased emphasis on searching for protective alleles [64], could provide new therapeutic opportunities to treat the over-nutritioned.

Satiety and energy expenditure hold the key to reversing the pandemic of over-nutritional phenotypes as a fundamental cure of symptoms manifested as obesity, diabetes, hypertension, cardiovascular disease, non-alcoholic steatohepatitis, cancer, depression, etc. Given the potential issues with drugs that result in satiety through attenuation of 'reward' pathways, e.g. increased incidence of depression and suicide with rimonabant, targeting energy expenditure may take centre-stage for the treatment of over-nutritional phenotypes. Importantly, for individuals beyond prevention, even moderate weight loss of $\sim 8 \mathrm{~kg}(17 \mathrm{lbs})$ had a profound impact on type 2 diabetics [65], yet most patients find sustaining weight reduction difficult. In animal models

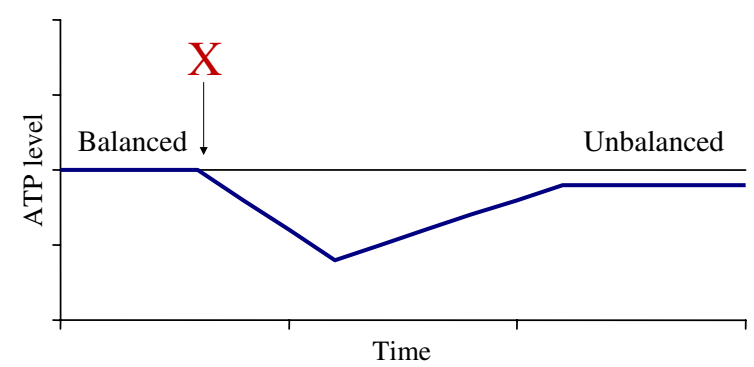

Fig. 5 Unifying theory: waste energy. There is no easy way to resolve an over-nutritional phenotype. However, it is clear that either energyin must be reduced or energy-out increased to reduce body weight. From an energy-out perspective, wasting energy by some mechanism is essential to take the body metabolically out of balance. A compound that wastes energy $(\mathrm{X})$ could, for example, do this by increasing urinary excretion of fuels (glucose, NEFA), releasing energy as heat, blocking intestinal absorption of potential fuels or by some as yet unknown mechanism. Whole-body energy-out has to be greater than energy-in and sustainable. The goal is to open the box for all possible disease-modifying therapies to realistically resolve the pandemic of over-nutritioned phenotypes. Blue line, energy (ATP level) 
in which $\dot{V} \mathrm{O}_{2}$ was shown to be increased this effect was repeatedly found to be 'disease modifying' [43, 66-69]. Insulin resistance, increased body weight, adiposity, steatosis, and elevated plasma lipids and cholesterol can be resolved by chronic increases in $\dot{V} \mathrm{O}_{2}$, suggesting a positive effect upon increasing energy expenditure. The significant hurdle in the search for novel drugs in this field is the identification of a mitochondrial uncoupler that is safe. However, given the potential benefits to patients and considering the pandemic of the over-nutritioned population, the endeavour is worth the effort.

Acknowledgements My sincere thanks to S. Black (Johnson \& Johnson PRD, Spring House, PA, USA) and D. Polidori (Johnson \& Johnson PRD, San Diego, CA, USA) for their helpful discussions and review of this perspective.

Duality of interest The author declares that there is no duality of interest associated with this manuscript.

\section{References}

1. Ntambi JM, Miyazaki M, Stoehr JP et al (2002) Loss of stearoylCoA desaturase-1 function protects mice against adiposity. Proc Natl Acad Sci USA 99:11482-11486

2. Sampath H, Flowers MT, Liu X et al (2009) Skin-specific deletion of stearoyl-CoA desaturase-1 alters skin lipid composition and protects mice from high-fat diet-induced obesity. J Biol Chem 284:19961-19973

3. Stone SJ, Myers HM, Watkins SM et al (2004) Lipopenia and skin barrier abnormalities in DGAT2-deficient mice. J Biol Chem 279:11767-11776

4. Ruderman NB, Saha AK, Kraegen EW (2003) Minireview: malonyl CoA, AMP-activated protein kinase, and adiposity. Endocrinology 144:5166-5171

5. Smith S, Witkowski A, Joshi AK (2003) Structural and functional organization of the animal fatty acid synthase. Prog Lipid Res 42:289-317

6. Coleman RA, Lee DP (2004) Enzymes of triacylglycerol synthesis and their regulation. Prog Lipid Res 43:134-176

7. Choi CS, Savage DB, Abu-Elheiga L et al (2007) Continuous fat oxidation in acetyl-CoA carboxylase 2 knockout mice increases total energy expenditure, reduces fat mass, and improves insulin sensitivity. Proc Natl Acad Sci USA 104:16480-16485

8. Abu-Elheiga L, Matzuk MM, Abo-Hashema KA, Wakil SJ (2001) Continuous fatty acid oxidation and reduced fat storage in mice lacking acetyl-CoA carboxylase 2. Science 291:2613-2616

9. Choi CS, Savage DB, Kulkarni A et al (2007) Suppression of diacylglycerol acyltransferase-2 (DGAT2), but not DGAT1, with antisense oligonucleotides reverses diet-induced hepatic steatosis and insulin resistance. J Biol Chem 282:22678-22688

10. Gutierrez-Juarez R, Pocai A, Mulas C et al (2006) Critical role of stearoyl-CoA desaturase-1 (SCD1) in the onset of diet-induced hepatic insulin resistance. J Clin Invest 116:1686-1695

11. Yu XX, Murray SF, Pandey SK et al (2005) Antisense oligonucleotide reduction of DGAT2 expression improves hepatic steatosis and hyperlipidemia in obese mice. Hepatology 42:362371

12. Harwood HJ Jr, Petras SF, Shelly LD et al (2003) Isozymenonselective N-substituted bipiperidylcarboxamide acetyl-CoA carboxylase inhibitors reduce tissue malonyl-CoA concentrations, inhibit fatty acid synthesis, and increase fatty acid oxidation in cultured cells and in experimental animals. J Biol Chem 278:37099-37111

13. Hoehn KL, Turner N, Swarbrick MM et al (2010) Acute or chronic upregulation of mitochondrial fatty acid oxidation has no net effect on whole-body energy expenditure or adiposity. Cell Metab 11:70-76

14. Hammond LE, Neschen S, Romanelli AJ et al (2005) Mitochondrial glycerol-3-phosphate acyltransferase-1 is essential in liver for the metabolism of excess acyl-CoAs. J Biol Chem 280:25629-25636

15. Nobes CD, Hay WW Jr, Brand MD (1990) The mechanism of stimulation of respiration by fatty acids in isolated hepatocytes. J Biol Chem 265:12910-12915

16. Abbott WC, Grakauskas AM, Bistrian BR, Rose R, Blackburn GL (1984) Metabolic and respiratory effects of continuous and discontinuous lipid infusions. Occurrence in excess of resting energy expenditure. Arch Surg 119:1367-1371

17. Dresner A, Laurent D, Marcucci M et al (1999) Effects of free fatty acids on glucose transport and IRS-1-associated phosphatidylinositol 3-kinase activity. J Clin Invest 103:253-259

18. Brand MD, Chien LF, Rolfe DF (1993) Control of oxidative phosphorylation in liver mitochondria and hepatocytes. Biochem Soc Trans 21(Pt 3):757-762

19. Boden G, Shulman GI (2002) Free fatty acids in obesity and type 2 diabetes: defining their role in the development of insulin resistance and beta-cell dysfunction. Eur J Clin Investig 32(Suppl 3): $14-23$

20. Rothwell NJ, Stock MJ (1979) A role for brown adipose tissue in diet-induced thermogenesis. Nature 281:31-35

21. Enerback S, Jacobsson A, Simpson EM et al (1997) Mice lacking mitochondrial uncoupling protein are cold-sensitive but not obese. Nature 387:90-94

22. Feldmann HM, Golozoubova V, Cannon B, Nedergaard J (2009) UCP1 ablation induces obesity and abolishes diet-induced thermogenesis in mice exempt from thermal stress by living at thermoneutrality. Cell Metab 9:203-209

23. Lodhi IJ, Semenkovich CF (2009) Why we should put clothes on mice. Cell Metab 9:111-112

24. Cannon B, Nedergaard J (2009) Thermogenesis challenges the adipostat hypothesis for body-weight control. Proc Nutr Soc 68:401-407

25. Koshkarian GM (1995) Congestive heart failure and sodium dichloroacetate. J Am Coll Cardiol 25:804-805

26. Foster DO, Frydman ML (1978) Nonshivering thermogenesis in the rat. II. Measurements of blood flow with microspheres point to brown adipose tissue as the dominant site of the calorigenesis induced by noradrenaline. Can J Physiol Pharm 56:110-122

27. Golozoubova V, Hohtola E, Matthias A, Jacobsson A, Cannon B, Nedergaard J (2001) Only UCP1 can mediate adaptive nonshivering thermogenesis in the cold. FASEB J 15:2048-2050

28. Heaton GM, Wagenvoord RJ, Kemp A Jr, Nicholls DG (1978) Brown-adipose-tissue mitochondria: photoaffinity labelling of the regulatory site of energy dissipation. Eur J Biochem $82: 515-521$

29. Nedergaard J, Golozoubova V, Matthias A, Asadi A, Jacobsson A, Cannon B (2001) UCP1: the only protein able to mediate adaptive non-shivering thermogenesis and metabolic inefficiency. Biochim Biophys Acta 1504:82-106

30. Christensen CR, Clark PB, Morton KA (2006) Reversal of hypermetabolic brown adipose tissue in F-18 FDG PET imaging. Clin Nucl Med 31:193-196

31. Virtanen KA, Lidell ME, Orava J et al (2009) Functional brown adipose tissue in healthy adults. N Engl J Med 360:1518-1525

32. Cypess AM, Lehman S, Williams G et al (2009) Identification and importance of brown adipose tissue in adult humans. $\mathrm{N}$ Engl J Med 360:1509-1517 
33. Lander ES, Linton LM, Birren B et al (2001) Initial sequencing and analysis of the human genome. Nature 409:860-921

34. Lou PH, Hansen BS, Olsen PH, Tullin S, Murphy MP, Brand MD (2007) Mitochondrial uncouplers with an extraordinary dynamic range. Biochem J 407:129-140

35. Christensen R, Kristensen PK, Bartels EM, Bliddal H, Astrup A (2007) Efficacy and safety of the weight-loss drug rimonabant: a meta-analysis of randomised trials. Lancet 370:1706-1713

36. Faulconbridge LF, Wadden TA, Berkowitz RI et al (2009) Changes in symptoms of depression with weight loss: results of a randomized trial. Obesity (Silver Spring) 17:1009-1016

37. Rolfe DF, Brand MD (1996) Contribution of mitochondrial proton leak to skeletal muscle respiration and to standard metabolic rate. Am J Physiol 271:C1380-1389

38. Stuart JA, Cadenas S, Jekabsons MB, Roussel D, Brand MD (2001) Mitochondrial proton leak and the uncoupling protein 1 homologues. Biochim Biophys Acta 1504:144-158

39. Leslie AG, Abrahams JP, Braig K et al (1999) The structure of bovine mitochondrial F1-ATPase: an example of rotary catalysis. Biochem Soc Trans 27:37-42

40. Tainter ML, Cutting WC, Stockton AB (1934) Use of dinitrophenol in nutritional disorders: a critical survey of clinical results. Am J Public Health Nations Health 24:1045-1053

41. Brand MD, Buckingham JA, Esteves TC et al (2004) Mitochondrial superoxide and aging: uncoupling-protein activity and superoxide production. Biochem Soc Symp 71:203-213

42. Korde AS, Pettigrew LC, Craddock SD, Maragos WF (2005) The mitochondrial uncoupler 2,4-dinitrophenol attenuates tissue damage and improves mitochondrial homeostasis following transient focal cerebral ischemia. J Neurochem 94:1676-1684

43. Caldeira da Silva CC, Cerqueira FM, Barbosa LF, Medeiros MH, Kowaltowski AJ (2008) Mild mitochondrial uncoupling in mice affects energy metabolism, redox balance and longevity. Aging Cell 7:552-560

44. Tainter ML, Stockton AB, Cutting WC (1935) Dinitrophenol in the treatment of obesity. J Am Med Assoc 105:332-337

45. Simkins S (1937) Dinitrophenol and desiccated thyroid in the treatment of obesity. J AMA 108:2110-2117

46. Gehring PJ, Buerge JF (1969) The distribution of 2,4dinitrophenol relative to its cataractogenic activity in ducklings and rabbits. Toxicol Appl Pharmacol 15:574-592

47. Olesen PH (2007) Novel compounds for the treatment of obesity. In. Novo Nordisk A/S, Bagsvaerd (DK), US 2007/0004799 A1, p 19

48. Hansen BS (2004) Chemical uncouplers for the treatment of obesity. In. Novo Nordisk Pharmaceuticals, Inc., US 2004/0138301 A1, p 61

49. Bellentani S, Marino M (2009) Epidemiology and natural history of non-alcoholic fatty liver disease (NAFLD). Ann Hepatol 8 (Suppl 1):S4-8

50. Bloomgarden ZT (2009) The 6th Annual World Congress on the Insulin Resistance Syndrome. Diab Care 32:e127-e133

51. Caccamo G, Bonura F, Vitale G et al (2010) Insulin resistance and acute coronary syndrome. Atherosclerosis 211:672-675

52. Kapur VK (2010) Obstructive sleep apnea: diagnosis, epidemiology, and economics. Respir Care 55:1155-1167

53. Li S, Zhao JH, Luan J, et al. (2010) Physical activity attenuates the genetic predisposition to obesity in 20,000 men and women from EPIC-Norfolk prospective population study. PLoS Med 7
54. Nguyen XM, Lane J, Smith BR, Nguyen NT (2009) Changes in inflammatory biomarkers across weight classes in a representative US population: a link between obesity and inflammation. J Gastrointest Surg 13:1205-1212

55. Osorio-Costa F, Rocha GZ, Dias MM, Carvalheira JB (2009) Epidemiological and molecular mechanisms aspects linking obesity and cancer. Arq Bras Endocrinol Metabol 53:213226

56. Sanchez-Villegas A, Pimenta AM, Beunza JJ, Guillen-Grima F, Toledo E, Martinez-Gonzalez MA (2010) Childhood and young adult overweight/obesity and incidence of depression in the SUN project. Obesity (Silver Spring) 18:1443-1448

57. Steyn NP, Mann J, Bennett PH et al (2004) Diet, nutrition and the prevention of type 2 diabetes. Public Health Nutr 7:147-165

58. Wilborn C, Beckham J, Campbell B et al (2005) Obesity: prevalence, theories, medical consequences, management, and research directions. J Int Soc Sports Nutr 2:4-31

59. Wiseman M (2008) The second World Cancer Research Fund/ American Institute for Cancer Research expert report. Food, nutrition, physical activity, and the prevention of cancer: a global perspective. Proc Nutr Soc 67:253-256

60. Wang L, Manson JE, Forman JP, Gaziano JM, Buring JE, Sesso HD (2010) Dietary fatty acids and the risk of hypertension in middle-aged and older women. Hypertension 56:598-604

61. King H, Rewers M (1993) Global estimates for prevalence of diabetes mellitus and impaired glucose tolerance in adults. WHO Ad Hoc Diabetes Reporting Group. Diabetes Care 16:157-177

62. Schulz LO, Bennett PH, Ravussin E et al (2006) Effects of traditional and western environments on prevalence of type 2 diabetes in Pima Indians in Mexico and the U.S. Diab Care 29:1866-1871

63. Katsuno K, Fujimori Y, Takemura Y et al (2007) Sergliflozin, a novel selective inhibitor of low-affinity sodium glucose cotransporter (SGLT2), validates the critical role of SGLT2 in renal glucose reabsorption and modulates plasma glucose level. J Pharmacol Exp Ther 320:323-330

64. Karelis AD (2008) Metabolically healthy but obese individuals. Lancet 372:1281-1283

65. Petersen KF, Dufour S, Befroy D, Lehrke M, Hendler RE, Shulman GI (2005) Reversal of nonalcoholic hepatic steatosis, hepatic insulin resistance, and hyperglycemia by moderate weight reduction in patients with type 2 diabetes. Diabetes 54 : 603-608

66. Cuevas-Ramos D, Almeda-Valdes P, Aguilar-Salinas CA, CuevasRamos G, Cuevas-Sosa AA, Gomez-Perez FJ (2009) The role of fibroblast growth factor 21 (FGF21) on energy balance, glucose and lipid metabolism. Curr Diabetes Rev 5:216-220

67. Gao Z, Yin J, Zhang J et al (2009) Butyrate improves insulin sensitivity and increases energy expenditure in mice. Diabetes 58:1509-1517

68. Li JZ, Ye J, Xue B et al (2007) Cideb regulates diet-induced obesity, liver steatosis, and insulin sensitivity by controlling lipogenesis and fatty acid oxidation. Diabetes 56:2523-2532

69. Pan D, Fujimoto M, Lopes A, Wang YX (2009) Twist-1 is a PPARdelta-inducible, negative-feedback regulator of PGC-1alpha in brown fat metabolism. Cell 137:73-86 